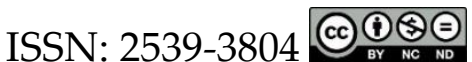

\title{
Prácticas de escritura en posgrado: el caso de una maestría en educación ${ }^{1}$
}

\author{
Héctor Iván Guerrero ${ }^{2}$ \\ Fundación Centros de Aprendizaje Neuroharte \\ Popayán, Colombia \\ Pilar Mirely Chois Lenis \\ Universidad del Cauca \\ Popayán, Colombia
}

\section{Resumen}

Este estudio etnográfico buscó describir las prácticas de escritura en una Maestría en Educación - modalidad investigación. Se encontró circulación de géneros discursivos escritos diversos, principalmente orientados a la evaluación de aprendizajes, y diferencias entre aquellos propuestos en la planeación y los solicitados realmente en clase. Aunque algunos docentes pretenden apoyar la escritura de los estudiantes, estos no siempre lo valoran positivamente, por la brecha que perciben entre las demandas en los seminarios y la urgencia de concluir el trabajo de grado. Parece necesario construir puentes entre las prácticas docentes sobre la escritura y las expectativas estudiantiles.

1 Este documento presenta los resultados del trabajo de grado requisito para optar al título de Magíster en Educación - Línea de Investigación Pedagogía de la Lectura y la Escritura, de la Universidad del Cauca. Se tituló Prácticas de escritura que promueven los profesores de la IV Cohorte de la Maestría en Educación de la Universidad del Cauca y contó con la participación de los estudiantes y profesores del programa. En su etapa inicial tuvo como directora a la profesora Pilar Mirely Chois Lenis y en la final, a la profesora Mary Edith Murillo Fernández. La financiación del estudio estuvo a cargo del investigador y fue llevado a cabo entre agosto de 2015 y junio de 2017.

2 Los autores agradecen a la Universidad del Cauca por ofrecer las condiciones para desarrollar este trabajo y a la profesora Mary Edith Murillo por su participación en la recta final de la investigación que dio origen a esta publicación. 
Palabras clave: práctica pedagógica; escritura; profesor; posgrado; maestría.

\begin{abstract}
Writing Practices at the Graduate Level: An Education Master's Degree Case

This ethnographical study was aimed to describe the writing practices within a Master in Education -research emphasis. We found several written discourse genres, mainly aimed to evaluate learning, and also differences between the writings proposed in the planning of the courses and those actually requested in class. Although professors try to support their students' writing, the latter usually do not consider it productive because of the perceived gap between what they are asked for in the seminars and their need to finish their monograph. It seems necessary to better connect the teaching practices on writing held by professors and the expectations from students in this particular program.
\end{abstract}

Key words: pedagogical practice; writing; professor; graduate; master.

\title{
Résumé
}

Les pratiques d'écriture dans un programme de master : le cas d'un master en Éducation

121 Cette étude ethnographique visait à interpréter les pratiques d'écriture dans un programme de master recherche en Éducation. Nous avons constaté la présence d'écrits s'inscrivant dans plusieurs genres de discours, notamment des textes voués à l'évaluation de l'apprentissage, et avons trouvé des distances entre les textes prévus dans le dessin des cours et les textes issus activités effectivement mises en place dans la salle de classe. Bien que les enseignants essaient de soutenir l'écriture de leurs étudiants, ceux-ci ne croient généralement pas que ce travail soit productif en raison du décalage entre ce que les enseignants demandent dans les séminaires et leur besoin de terminer leurs travaux de fin d'études. Il s'avère donc important de relier les pratiques d'écriture proposées par les professeurs et les attentes des étudiants.

Mots-clés : pratique pédagogique ; rédaction ; Professeur ; Postgraduate ; Master. 


\section{CóMO CITAR ESTE ARTÍCULO}

Guerrero, H. I., y Chois Lenis, P. M. (2019). Prácticas de escritura en posgrado: el caso de una maestría en educación. Lenguaje, 47(1), 120-146. doi: 10.25100/lenguaje.v47i1.6094 


\section{INTRODUCCIÓN}

La relevancia que la escritura en posgrado ha ido ganando en Latinoamérica puede justificarse por ser considerada una condición necesaria para la construcción del conocimiento (Carlino, 2005; Scardamalia y Bereiter, 1992), para el ingreso a una comunidad disciplinaria (Amieva y De Angelo, 2010) y por su imbricación con la investigación (Carlino, 2005; Miras y Solé, 2007).

Por eso, la escritura se ha asumido como objeto de investigación e intervención pedagógica en posgrados de Latinoamérica desde inicios de este siglo. Varios estudios se han concentrado en evaluar el desempeño de los estudiantes, encontrando que tienen problemas para revisar sus propios textos (Carlino, 2005), para reconocer los géneros discursivos propios de este nivel (Sabaj, 2009), para conectar la información (Castelló, González e Iñesta, 2010), para diferenciar las voces presentes en el texto (Aguilar y Fregoso, 2013) y hasta para empezar a escribir (Cruz Martínez, 2014).

Sin embargo, una perspectiva social de la escritura permite reconocer que no se trata de un saber que se tenga o no gracias a habilidades individuales exclusivamente, sino de una práctica social que se construye y transforma durante la participación en prácticas discursivas de una comunidad. Eso justifica que los programas de posgrado se interesen por promover entre los aprendices la apropiación de las prácticas discursivas propias de las comunidades disciplinarias en las que están incursionando.

En el ámbito internacional, por ejemplo, se han documentado algunas experiencias pedagógicas orientadas en ese sentido (por ejemplo: Álvarez y Difabio de Anglat, 2017; Carlino, 2005; Colombo, 2017; Fernández y Guevara, 2017; Narvaja de Arnoux, 2010). Sin embargo, de acuerdo con Chois y Jaramillo (2016), en Latinoamérica son escasos los estudios que focalicen la práctica pedagógica sobre la escritura en posgrado.

Además, pocos trabajos han explorado específicamente las prácticas de docentes especialistas en un campo disciplinario que no corresponde a la lectura y la escritura, prácticas que no necesariamente están orientadas a enseñar a escribir, pero pueden dar cuenta de las maneras de leer y escribir específicas de la formación en un área del saber.

Aquí se presentan los hallazgos de una investigación que tuvo como objetivo describir las prácticas de escritura que promueven los profesores de la Maestría en Educación de una Universidad Pública del suroccidente colombiano. El propósito es aportar, por un lado, a la reflexión de docentes y directivos interesados en la formación escritural en posgrado; y, por otro lado, a la configuración del campo de la escritura académica y disciplinaria en la región. 


\section{REFERENTE TEÓRICO}

\section{Escritura como práctica social}

Pereira y Di Stefano (2007) señalan que los enfoques que conciben la escritura como práctica social provienen de distintos campos disciplinarios, como la psicología social, el análisis del discurso, la sociología y la historia social de la lectura y la escritura.

Según Barton y Hamilton (2004), la escritura es una práctica inseparable de los contextos sociales y culturales en los que tiene lugar, es decir, se trata de una práctica condicionada social e históricamente. Se lleva a cabo de formas particulares por los miembros de una determinada comunidad, quienes conocen y ejercen sus formas -géneros, estilos, formatos discursivos- y contenidos de comunicación -temas, conceptos y teorías-, de acuerdo con sus propósitos y con las relaciones de poder e identidad que entre ellos reconocen (Hernández Zamora, 2009). Analizar la escritura como práctica, plantean Pereira y Di Stefano (2007), permite focalizar el modo en que se construye socialmente el sentido, así como los elementos que hacen posible y significativa su circulación.

Por ello, se reconoce que los miembros de una comunidad específica comprenden y producen el discurso que en ella se genera, lo cual los convierte en sujetos de participación activa. En consecuencia, asuntos como los contenidos, objetivos, formas y públicos a quienes se dirige un documento se aprenden participando en las prácticas propias de un contexto determinado (Cassany y Morales, 2009).

Así, para Colombo (2012b, 2017), una perspectiva social de la escritura destaca la estrecha relación entre la escritura y el contexto disciplinario en el cual se ejerce, así como la relevancia de las relaciones sociales en las prácticas letradas para su aprendizaje. Por lo tanto, considera que los estudiantes de posgrado pasan a integrar las comunidades disciplinarias de práctica, interactuando con otros y produciendo textos para otros. Coinciden con ella Guerrero y Mostacero (2014), al señalar que la interacción en una comunidad académica surge en situaciones discursivas concretas y reales, en el interior de prácticas situadas en un contexto particular. Eso explica para estos autores que, si tales condiciones no se dan durante la producción del trabajo de grado, el estudiante no logra identificarse con sus pares. 


\section{Los géneros discursivos en posgrado}

Miller (1984) reconoce como enfoques de estudio de los géneros: a. estudios de géneros literarios desde la teoría literaria, la teoría cinematográfica y otras teorías culturales; b. estudios de género lingüístico desde la Lingüística Funcional Sistémica o "SFL", c. estudios de género literario desde la escuela del inglés para fines específicos o "ESP" y d. los estudios de Género Retórico o "RGS", que estudian el género como acción social.

Para este trabajo partimos de una mirada centralmente lingüística, que reconoce los géneros discursivos como artefactos con rasgos lingüísticos estables, que circulan en una comunidad determinada. Así, desde Bajtín (1982), asumimos los géneros como la forma de construcción de enunciados de cada esfera del uso de la lengua, que se encuentra demarcada por la riqueza de éstos, en razón a las relaciones fundadas en la complejidad.

Desde esta perspectiva, se entienden los géneros discursivos escritos como tipos de textos que se caracterizan por rasgos comunes tales como su organización global, así como por normas convencionales de producción, interpretación y circulación. Es decir, se reconocen como sistemas de acción que generan expectativas entre autores y lectores (Carlino, 2005).

Preguntarse por los géneros discursivos propios de la formación en posgrado implica reconocer las comunidades y textos que en ese ámbito tienen lugar. Russell y Cortes (2012) discuten sobre los calificativos académico y científico empleados usualmente para referirse a la escritura en la universidad. Se adscriben a la postura según la cual la comunidad científica está compuesta por las personas que en su ejercicio aportan y se alimentan de la disciplina.

En ese sentido, entienden la escritura académica como aquella producida por estudiantes para responder a los compromisos derivados de su formación universitaria, y la escritura científica como la producida por los investigadores con el propósito de comunicar conocimiento nuevo. Sin embargo, Russell y Cortes (2012) reconocen que ambos ámbitos se interrelacionan en términos de desarrollo intelectual y psicológico, así como en términos de categorías institucionales.

Desde esta perspectiva puede comprenderse que, en los estudios de posgrado, más aún en los programas orientados a la investigación, se lean y escriban géneros discursivos tanto académicos como científicos. 


\section{REFERENTE CONTEXTUAL}

En Colombia, el posgrado corresponde al máximo nivel de educación superior y está conformado por especialización, maestría y doctorado, en orden de menor a mayor jerarquía (Ministerio de Educación Nacional, 1980).

Específicamente los estudios de maestría se ofrecen en dos modalidades: profundización e investigación. La primera tiene como propósito la profundización en áreas específicas y el perfeccionamiento de competencias para la solución de problemas a través de la apropiación de conocimientos, metodologías y desarrollos. La segunda, en tanto, pretende el desarrollo de competencias para la participación activa en procesos de investigación, orientados a generar nuevo conocimiento (Ministerio de Educación Nacional, 2006).

Este estudio se llevó a cabo en un Programa de Maestría en Educación, modalidad investigación, creado en el año 2005, que según su documento maestro tiene el objetivo de: "contribuir a la formación de educadores capaces de abordar, mediante la investigación, problemáticas de su campo profesional desde una perspectiva teórica y metodológica investigativa que les permita diseñar, desarrollar y sistematizar proyectos educativos, comunitarios y pedagógicos" (Maestría en Educación, 2007, p. 16).

Respondiendo a principios como la integración, la interdisciplinariedad, la pertinencia y la flexibilidad, el Programa contempla dos componentes de formación. El primero es la fundamentación teórica, que se desarrolla en los seminarios que abordan la educación y la investigación educativa como temas centrales. El segundo es el de investigación, construido alrededor de la actividad indagadora de los participantes a través de la consolidación de sus proyectos de investigación.

En coherencia con lo anterior, la Maestría ofrece seminarios: a. de fundamentación teórica, $b$. de línea de investigación, c. de integración y d. electivos. Los de fundamentación teórica y los de integración son obligatorios para los inscritos en el Programa, mientras que los de línea son cursados por los maestrantes de acuerdo con la línea en la que fueron aceptados. Se ofrecen seis líneas denominadas: a. Educación multicultural y Etnoeducación; b. Motricidad y desarrollo humano, c. Saber Pedagógico, d. Pedagogía de la lectura y la escritura, e. Comunicación, Educación y Cultura y f. Enseñanza de las ciencias y la Tecnología.

En cada uno de los cuatro semestres en que los estudiantes cursan seminarios, se incluye uno de línea, en los que se formen como investigadores en la especificidad del campo del saber en el que se inscriben. En los seminarios de integración, por su parte, socializan los avances de su proyecto de investigación ante diferentes profesores y compañeros. 
Además de los aportes dados por estos seminarios, cada estudiante cuenta, desde tercer semestre, con un director de trabajo de grado. Este trabajo, requisito obligatorio para la titulación, se entiende según el documento maestro del Programa como "un aporte teórico o práctico a la ciencia o a sus aplicaciones" (Maestría en Educación, 2007, p. 35).

\section{Metodología}

Esta investigación asume una metodología cualitativa con enfoque etnográfico. Se recabó información durante los cuatro periodos académicos en que se desarrollaron los seminarios ofrecidos a la IV cohorte de la Maestría en Educación, entre 2015 y 2017, mediante cinco técnicas de recolección: observación participante, análisis documental, encuesta, grupo de discusión y entrevista.

La observación participante en cuatro Seminarios de la Línea de Pedagogía de la Lectura y la Escritura, cuatro Generales y cuatro Electivos permitió la exploración directa de las prácticas docentes in situ de los nueve profesores que los orientaron.

Por otra parte, el análisis documental, como técnica de recolección de información (Goetz y LeCompte, 1988), posibilitó acceder a las prácticas docentes planeadas, mediante la indagación de los microcurrículos o programas de los seminarios socializados y entregados a los estudiantes desde el inicio de cada periodo académico.

También se solicitó a los 27 estudiantes que se encontraban cursando todos los seminarios de la IV cohorte responder, a través del aplicativo Google Forms, una encuesta en línea, orientada en primer lugar a caracterizar esta población -en cuanto a sus lugares de trabajo, experiencia laboral, área del conocimiento y programa académico del que provienen- $\mathrm{y}$, en segundo lugar, a explorar la forma en que los estudiantes interpretaban la labor de los profesores -en cuanto al fomento de prácticas de escritura, los apoyos que ofrecían durante ellas, los momentos empleados para tal fin y los resultados esperados/alcanzados-. Allí, además, se les solicitó mencionar un profesor que, según su criterio, pudiera ser reconocido por apoyar la escritura de los maestrantes.

Con el objeto de indagar en algunos temas revelados durante la encuesta, se llevó a cabo un grupo de discusión (Corbetta, 2003) con cinco estudiantes que representaban ambos sexos, todas las líneas de investigación y diferentes momentos de desarrollo del trabajo de grado. Con base en un derrotero de preguntas orientadoras, resultado de la matriz de categorías iniciales, el grupo de discusión estuvo dirigido a explorar la forma en que los estudiantes respondían a 
las prácticas de escritura que fomentaban sus profesores, y las maneras en que las interpretaban.

Por último, se hizo una entrevista semiestructurada al profesor más mencionado por los estudiantes en la encuesta, como aquel que apoyaba su escritura, mediante un instrumento construido por el investigador y avalado por pares expertos. La entrevista tuvo por objeto explorar las prácticas referidas por el docente respecto a la escritura de sus estudiantes y su interpretación de dichas prácticas.

La información recolectada a través de las diferentes técnicas fue registrada en audio y video, para después ser seleccionada, transcrita y finalmente analizada. Toda la información fue codificada durante su proceso de organización y análisis, tal como se muestra en la Tabla 1. Estos códigos son usados durante el artículo para dar cuenta de la fuente de la información usada como evidencia empírica.

Tabla 1. Código de los datos según técnica y población

\begin{tabular}{|c|c|c|}
\hline TÉCNICA & $\begin{array}{l}\text { FUENTE DE } \\
\text { INFORMACIÓN }\end{array}$ & CÓDIGO \\
\hline \multirow{3}{*}{$\begin{array}{l}\text { Observación directa } \\
\text { Seminario-tipo-\#-fecha }\end{array}$} & Seminario General & O SG\# fecha observación \\
\hline & Seminario Línea & O SL\# fecha observación \\
\hline & Seminario Electivo & O SE\# fecha observación \\
\hline Entrevista & $\begin{array}{l}\text { Profesor } \\
\text { Entrevista-profesor-informante-\#- } \\
\text { fecha }\end{array}$ & E P Inf1 fecha \\
\hline Encuesta & $\begin{array}{l}\text { Estudiantes } \\
\text { Encuesta-informante-estudiante-\#- } \\
\text { fecha }\end{array}$ & E Infe \# fecha \\
\hline Grupo de discusión & Estudiantes & GD Infe1-5 fecha \\
\hline \multirow[t]{3}{*}{ Revisión documental } & Programa Seminario General & P SG\# \\
\hline & Programa Seminario Línea & P SL\# \\
\hline & Programa Seminario Electivo & P SE\# \\
\hline
\end{tabular}

Fuente: Elaboración propia

El análisis de la información recolectada permitió la consolidación de la matriz de categorías definitivas. Para el análisis de la información, se consideraron 
como categorías iniciales las prácticas de escritura, los estudios de posgrado y los profesores de maestría, lo que orientó, por un lado, la búsqueda de información y, por el otro, su posterior organización y análisis.

\section{Población de estudio}

De los nueve profesores que orientaron los seminarios observados, cinco son de género masculino. Todos cursaron sus estudios de pregrado en universidades públicas colombianas en las áreas de Licenciatura (siete profesores), antropología (uno) y estadística (uno). Los nueve docentes cuentan con título de maestría, seis de ellos con título de doctorado y uno lo estaba cursando durante el registro de la información.

De los 27 estudiantes de la IV cohorte, 19 respondieron la encuesta. El $61 \%$ correspondía al género femenino; el 44,4\% de ellos son egresados de programas de las Ciencias Humanas y Sociales; el 27,7 \%, de Ciencias de la Educación; 11,5\%, de Ingenierías; 5,5\%, de Bellas Artes, y 5,5\%, de las Ciencias de la Salud. El $83 \%$ de ellos se desempeñan como docentes de aula adscritos a la entidad territorial en la que se desarrolló el estudio y los demás, en instituciones educativas de carácter 129 privado.

\section{Aspectos éticos y legales}

Una vez se presentaron las solicitudes institucionales correspondientes, se obtuvo la autorización de la Decanatura de la Facultad a la que está adscrito el Programa académico objeto de estudio y de su Coordinación.

Además, todos los profesores y estudiantes consultados aceptaron participar en el estudio mediante la firma del Consentimiento Informado, en el que se les presentó la información básica de esta investigación y se les planteó que la información recolectada sería usada con fines académicos, bajo los principios de confidencialidad y protección de los datos.

\section{ANÁlISIS Y DISCUSIÓN DE RESULTADOS}

Se presentan a continuación algunos hallazgos organizados en cuatro subapartados acerca de las prácticas de escritura en el Programa de Maestría en Educación modalidad investigación denominados: a) Los géneros discursivos escritos en la Maestría en Educación, b) Los pre-textos de escritura académica, c) Actuaciones docentes para apoyar la escritura académica y d) Relación entre escritura en los seminarios y proyecto de investigación. 


\section{Los géneros discursivos escritos en la Maestría en Educación}

De acuerdo con los apartados de metodología, evaluación y objetivos de los programas de cada uno de los seminarios, se observó que los profesores planearon proponer a los maestrantes leer géneros discursivos que denominaron artículo científico/académico, material de trabajo elaborado para la sesión, libro y resumen. Además, anticiparon proponer la escritura de géneros discursivos que denominaron artículo, resumen, resumen analítico especializado (RAE), análisis de lectura, protocolo, ensayo, trabajo escrito, reseña, portafolio multitextual, anteproyecto, apartado de trabajo de grado, informe final de investigación, instrumento de recolección de información (diario de campo), relatoría, correlatoría, cartelera, cuestionario, informe de lectura, reflexión crítica, valoración sociocrítica y matriz.

Puede observarse que son muchos más los géneros discursivos que se planea proponer escribir a los maestrantes que aquellos que se planea proponerles leer. Algunos de los géneros mencionados en los programas o microcurrículos, tales como resumen, ensayo, reseña y trabajo de grado, son referidos en la bibliografía como comunes de los niveles de posgrado (Amieva y De Angelo, 2010; Colombo y Zambrano, 2013; Horowitz, 1986; Pereira y Di Stefano, 2007).

Sin embargo, algunos de los mencionados en los programas de los seminarios, realmente no se hicieron presentes durante el desarrollo de las clases. Tal es el caso del artículo, género discursivo sugerido como material de lectura en todos los seminarios, pero no solicitado como producto escrito a los estudiantes, como estaba previsto en los microcurrículos de dos seminarios generales y dos de énfasis.

La inclusión del artículo como género que debe ser leído o escrito por los estudiantes de maestría, sobre todo de aquellas con énfasis en investigación, está justificada porque reviste gran importancia en los medios académicos (Colombo, 2012a; Swales, 1990) y porque autores como Day (2005) consideran que una investigación solo culmina con la publicación de sus hallazgos. Además, uno de los aspectos por considerar durante el proceso de evaluación con fines de acreditación de los programas académicos de posgrado son las publicaciones y otros productos de los grupos de investigación. Con la intención de promover esa práctica, el último seminario de integración de la maestría se titula Publicación y socialización del informe final del trabajo de grado. No obstante, la publicación de artículos como mecanismo para divulgar los hallazgos investigativos entre la comunidad científica no se promueve consistentemente en la maestría. Se trata más bien de una práctica que depende de las decisiones particulares de los directores de trabajo de grado y sus dirigidos. 
En coherencia con los hallazgos de este estudio, Colombo y Zambrano (2013) encontraron en distintas facultades de una universidad norteamericana que se trata de un género discursivo escasamente solicitado, según la información aportada por los estudiantes.

Otros géneros discursivos escritos se destacan en este estudio porque, a pesar de ser demandados solo en dos seminarios (SG2; SE1), se encontró que los profesores ofrecieron apoyos a los estudiantes para su elaboración.

Por ejemplo, se solicita la escritura de RAE, género discursivo que implica la lectura de un artículo de investigación publicado y la elaboración de una síntesis de dicho artículo, usualmente en una tabla con ítems establecidos por el docente, y que difieren según quien lo demande. Algunos solicitan explicitación del tema, objetivos, conceptos e ideas centrales, resumen y conclusión (O SE3 18022017), en tanto otros piden señalar el tipo de texto, título, autor, palabras clave, descripción, fuente, contenidos y comentarios (O SG2 15112015). Puede identificarse que el segundo tipo exige al estudiante hacer un aporte personal a los planteamientos del texto, en tanto el primero no.

La demanda de este género representa para dos de los profesores, observados directamente en clase, la necesidad de ofrecer un modelo por seguir a 131 sus estudiantes. Por ello, entregan a los maestrantes un RAE ya elaborado, el artículo de investigación sobre el cual deben elaborar el nuevo RAE -no aquel sobre el que fue elaborado el RAE entregado como modelo-, y solicitan tomar como referencia el primero para la elaboración del segundo.

Si bien puede ser útil para los estudiantes contar con un modelo por seguir, no necesariamente es suficiente ver un texto ya elaborado para ser capaz de elaborar otro con similares características, más aún si el modelo es solo entregado y no se convierte en objeto de análisis guiado.

No se observó la enseñanza explícita de las características de este género discursivo, práctica recomendada por autores como Carlino (2009) y Colombo (2016). Es importante destacar que la bibliografía revisada no hace referencia al RAE específicamente; sin embargo, Mostacero (2013) documentó un trabajo que orientó en una maestría en Lingüística, sobre la reseña crítica. Este género, de acuerdo con su descripción, comparte algunas características con el RAE tal como se demanda en algunos casos observados. Por ejemplo, ambos incluyen en el cierre apartados tales como conclusión y comentarios, que implican una reflexión crítica de parte de los autores frente a los aportes del documento leído.

Otro género al que algunos docentes otorgaron especial interés en el Programa fue el Protocolo, ya que tanto sus características como su definición fueron ampliamente explicitadas en clase y en los documentos guía entregados para su elaboración. Cada uno de los profesores dedicó varias clases a apoyar su construcción, mediante la revisión de borradores y asesoría a los autores. 
Durante una de las sesiones en la que el profesor hizo lectura del Programa del seminario general a su cargo, planteó como definición:

el protocolo es un documento público, por lo que debe ser dirigido con cuidado. Abrir el debate, coger la tesis y desarrollarla, tener en cuenta acuerdos y desacuerdos presentados de forma académica. Trasciende, reconstruye y recrea. Da lugar a pensamientos del autor del seminario $(O$ SG1 2082015).

Aquí se destaca el papel de quien lo elabora, encargado de recoger la pluralidad de voces durante la clase y dar cuenta de su propia voz al respecto. De acuerdo con lo que explica un docente en clase: "El protocolo es el sofoco de la reflexión, abre la participación. (...) No es un amaño del protocolante, el protocolante saca la discusión del remolino que se ha creado, para darle continuidad a la discusión" (O SG1 21082015).

Implica, además, según plantea un profesor durante una clase del seminario a su cargo, el concurso de otros materiales bibliográficos: "Es un documento reflexivo que abre los horizontes expansivos de análisis; se debe utilizar otros documentos para complementar" (O SG1 21082015).

En otro de los seminarios, se lo enuncia como un producto escrito que, al inicio, recoge fielmente lo ocurrido en la clase. En este caso, se pide a los estudiantes considerar los momentos en que se organiza la sesión, para luego asumir una posición que le permita juzgar y evaluar su desarrollo desde una posición crítica, dando lugar a un producto que, además de informar sobre lo sucedido, implique tomar distancia de ello:

Es el producto de cada una de las sesiones del Seminario Investigativo. Deberá recoger fielmente lo ocurrido en la sesión. El autor del Protocolo, o Protocolante, deberá juzgar, evaluar el desarrollo de la sesión, tomando posición crítica. Debe plasmar su aporte, ya que una vez terminada la sesión dispondrá de tiempo suficiente para procesar lo ocurrido y a través de la reflexión sobre el proceso integral de la sesión (relatoría, correlatoría, discusión y dirección), podrá esclarecer sus acuerdos y desacuerdos (P SG3).

Por otro lado, el resumen, análisis y control de lectura, mencionados por Colombo y Zambrano (2013) como los géneros más solicitados en los posgrados de la facultad de humanidades donde realizaron su estudio, aparecen en esta investigación como géneros discursivos sugeridos o recomendados por los profesores. No obstante, estos no exigen su presentación de manera obligatoria y, dado que no son objeto de calificación, los estudiantes deciden si elaborarlos o no. 
Estos denominados "trabajos escritos" se caracterizan en el Programa por no tener una superestructura, extensión o parámetros estrictamente definidos por parte de los profesores.

La solicitud de estos géneros discursivos parece estar orientada a facilitar la apropiación de los contenidos, es decir, los profesores sugieren la construcción del resumen, análisis y control de lectura cuando, a su parecer, han solicitado leer textos de alta complejidad. En otros casos, la solicitud de estos géneros se encuentra relacionada con la importancia que otorga el profesor a la necesidad de que sus estudiantes preparen previamente la temática por trabajar, y con el tiempo disponible, tal como lo refiere uno de los profesores durante una de sus clases y uno en el Programa del seminario a su cargo:

(...) para que nos rinda, porque tenemos mucho que avanzar, aunque vamos bien en el cronograma, deben traer leído todo el artículo, que es lo que nos va a llevar a la siguiente actividad. Lean con mucha atención y escriban sus dudas o sus claridades (O SE3 18022017).

La mayor parte de los temas comenzarán con una lectura (realizada por el estudiante (en casa) de uno o varios textos asociados al tema y finalizará con un pequeño ejercicio escrito (P SG 2).

Así, en la Maestría en Educación, usualmente el resumen, análisis y control de lectura no son revisados ni calificados por parte de los profesores, razón por la cual durante el grupo de discusión solo un estudiante afirmó haberlo hecho cuando se lo sugirieron y otro reconoció no hacerlo porque nadie más lo hacía: "nadie iba a ponerse más trabajo, si de eso ni nota sacaban. Además, yo creo que en eso todos estábamos de acuerdo" (INFE 4 08042017). Algunos profesores, no obstante, les piden apoyarse en sus tareas durante la discusión en clase.

Para finalizar, los denominados "trabajos escritos" se solicitan en la totalidad de los seminarios ofrecidos en el Programa académico investigado, sin explicitar necesariamente a los maestrantes las características de los productos que deben presentar. Dicha situación podría explicarse por parte de los profesores como una invitación a la autonomía de parte de los estudiantes, referida por Gaviria, Rincón y Triana (2016).

Se encontró que los profesores solicitan los llamados trabajos escritos principalmente como requisito final para acreditar los seminarios, mediante la asignación de una calificación. Los estudiantes rechazan esa práctica alegando que "por lo menos deberían explicarnos para qué sirve (el trabajo) aparte de la nota, y sobre todo por qué siempre es la misma (nota) para todos" (GD INFE 2 08042017). En este sentido, el trabajo escrito se caracteriza por no tener características ni 
denominaciones unificadas, y se solicita en clase como ensayo, reflexión, valoración y hasta resumen.

\section{Los pre-textos de escritura académica}

Entendiendo la escritura como una práctica social desarrollada por los miembros de una comunidad con propósitos particulares, exploramos las razones o causas aparentes, por las cuales se demandó escribir a los estudiantes en la maestría, lo que constituye la categoría pre-textos de escritura académica que se presenta a continuación.

A lo largo de la investigación se encontró que el momento escogido por los profesores para la solicitud de producciones escritas es predominantemente el final de un seminario, de una actividad o de una temática. Solo en algunas ocasiones se hace esta solicitud en momentos diferentes, y lo usual es que los autores no reciban realimentación ni calificación, cuando la actividad no corresponde al trabajo final del seminario. Así lo manifestó un estudiante en el grupo de discusión: "se entregaron como seis RAE, pero yo no creo que (el profesor) ni los haya leído, supuestamente los iba a devolver, pero yo sigo esperando" (GD INFE 4 08042017).

En consecuencia, para los estudiantes, la razón que motiva la solicitud de textos escritos en la maestría es predominantemente la obtención de calificaciones, para aprobar o no un seminario.

$\mathrm{Al}$ respecto, el docente entrevistado acepta que eso es posible y lo cuestiona:

esa preocupación de que un estudiante entregue un trabajo... pero la pregunta sería qué tanto el profesor se preocupa por leer ese trabajo del estudiante, porque la escritura tiene sentido si hay un interlocutor que me va a leer. Cuando yo cumplo con entregar un documento escrito, pero que no tengo la garantía de retroalimentación del documento escrito, pues eso no me exige escribir... y yo puedo escribir cualquier cosa (E P INF1 270517).

Es importante destacar entonces que el cumplimiento de este tipo de deberes termina siendo para los estudiantes, en línea con lo referido por Moreno Bayardo (2011), una sobrecarga de trabajo. Por ello se quejan del exceso de actividades y proponen minimizar las tareas extra clase, tal como lo expresa un estudiante durante una sesión de seminario: “...ustedes dejan las copias, el libro, el correo, pero en la semana uno tiene otras obligaciones, entonces por qué no hacer todo aquí en la clase" (O SG2 15112015). Además, siempre aceptan la propuesta de sus profesores o ellos mismos proponen hacer, en lugar del trabajo escrito solicitado desde el inicio del seminario, otro tipo de producciones, tales como obras de teatro que los exime de la tarea de escribir. 
En algunas ocasiones, los planteamientos incluidos en los microcurrículos de los seminarios permiten revelar que los profesores atribuyen a la escritura un papel distinto al de calificar al estudiante, en tanto refieren como uno de los objetivos de estas actividades la adquisición de conocimientos. Así se evidencia en un Programa de seminario: "Alcanzar los objetivos propuestos para el seminario, implica asumirlo también como un espacio de lectura y escritura, en reconocimiento de estos procesos como medios privilegiados para la construcción del conocimiento" (P SL3). Este fragmento alude al potencial epistémico de la escritura, es decir, a la posibilidad de escribir como herramienta para transformar el propio conocimiento (Carlino, 2009).

\section{Actuaciones docentes para apoyar la escritura académica}

Las actuaciones desarrolladas por los docentes para apoyar la escritura académica de sus estudiantes en la Maestría en Educación fueron rastreadas, por un lado, en los microcurrículos que entregaron al inicio de los seminarios y por el otro, durante su desarrollo. Se encontró que en ocasiones se plantean de forma explícita en los programas de seminario $\mathrm{y}$, en otras oportunidades, surgen en la dinámica de la clase de manera no necesariamente intencional.

Entre esas actuaciones pueden identificarse: lectura (a veces comentada) durante las clases, asesorías virtuales o presenciales, trabajos en grupo orientados a la producción de textos escritos, análisis grupal de textos académicos, entrega de instructivos, presentación de modelos de textos escritos, entrega de guías de lectura y revisión de borradores.

Algunas de las actuaciones anticipadas en los microcurrículos no se llevan a cabo realmente durante las clases, por cuestiones de tiempo o por la misma exigencia de los estudiantes, que afirman sentirse desbordados por las demandas y apoyos ofrecidos por sus profesores, pero que ellos entienden como una obligación más por cumplir: "entonces empiezas a escribir, o a preparar lo que quieres presentar, pero sabes que deberías estar haciendo el anteproyecto, porque ahí tienes al otro (al director de tesis) que está encima de eso" (GD INFE 2 08042017).

Es notoria la brecha entre la propuesta de los profesores y la interpretación que de ella hacen los estudiantes. Por ejemplo, ante las observaciones y comentarios escritos que hacen algunos profesores en los textos de sus estudiantes, con el propósito de ayudarles a cualificarlos, se encuentran apreciaciones estudiantiles como "(...) yo sí prefiero que no me escriban nada, que jartera la 
rayadera, sobre la rayadera ${ }^{3}$ es como si no quisieran que terminemos eso nunca" (GD INFE 1 08042017).

Esto coincide con los hallazgos de Moreno Bayardo (2011), según los cuales a pesar de que muchos profesores intentan ofrecer apoyos para sus estudiantes, estos no siempre los comprenden y en ocasiones llegan a considerarlos como circunstancias adversas a su proceso formativo.

Otra actuación docente que podría apoyar la escritura de los estudiantes es proponerles realizar grupalmente trabajos que implican la realización de un texto escrito, pues se convierte en una oportunidad para el diálogo y la construcción conjunta de conocimiento. Usualmente se propone para la realización de exposiciones o trabajos finales, y de ello podría inferirse un interés del Programa por fomentar la dinámica de trabajo propia de los grupos de investigación.

Al respecto, aunque según los documentos oficiales de la maestría, este programa está sustentado en grupos de investigación, las propuestas para los proyectos de grado surgen predominantemente de intereses particulares de los estudiantes, no necesariamente ligados a las dinámicas de los grupos. Así lo planteó un estudiante en el grupo de discusión:

con lo de los macroproyectos (proyectos docentes que incluyen a varios maestrantes), todos queríamos eso porque era más fácil. Pero nadie, creo que una línea sí, el resto todos hicimos el tema que traíamos. Bueno, o lo que quedaba de eso (GD INFE 4 08042017).

Así las cosas, en pocas oportunidades el estudiante se integra a un proyecto de investigación docente o participa en un grupo de investigación, a pesar de que trabajos como el realizado por Pereyra y Cardoso (2017) reconocen la importancia de hacerlo. Trabajos como los de Carrasco, Kent y Keranen (2012) y Delamont y Atkinson (2001) también han documentado el soporte que significa para la escritura en posgrado el que los aprendices participen activamente en colectivos integrados por investigadores de diferente nivel de experticia.

Por otra parte, algunos programas de seminario proponen la discusión como actividad simultánea, de apoyo o complemento para los trabajos en grupo, sobre la base de que socializar dudas o claridades entre los integrantes ayuda a mejorar la comprensión del tema de estudio. Este hecho cobra relevancia en la escritura académica, en tanto los diferentes trabajos en grupo son evaluados por medio de un producto final que usualmente es escrito.

\footnotetext{
${ }^{3}$ Expresión que hace alusión a los múltiples comentarios y marcas que hacen los docentes sobre los textos escritos por los estudiantes.
} 
Todos los profesores, en las clases registradas por medio de la observación participante y en los microcurrículos, dan cuenta de la importancia que otorgan a poner en diálogo los temas abordados durante las sesiones, a través de clases magistrales o lecturas de apoyo, con los saberes previos de los estudiantes, que a través de sus experiencias propician espacios de reflexión, en la mayoría de los casos bajo el arbitraje de los profesores.

Aunque los espacios de discusión son referidos por todos los profesores durante sus clases y en los microcurrículos como una oportunidad para dialogar y compartir saberes a partir del trabajo colaborativo y multidisciplinario, para los estudiantes los ejercicios de discusión representan sesiones de trabajo extenuantes que no ofrecen una posibilidad académica sino más bien un espacio para compartir experiencias docentes y estudiantiles que no aportan a la construcción del producto final escrito.

Así lo manifiestan dos participantes en el grupo de discusión:

(...) uno lee, uno intenta tomar como sus propias notas de lo que entiende. Pero escuchar a otras personas que tampoco están seguras que entienden, eso es como un poco desmoralizante (GD INFE 4 08042017).

(...) pues cuando se ponen a hablar y hablar, y hablan de todo. Pasan de física a matemáticas, luego de desplazados, hasta sexualidad y uno al final ¿de qué se coge?, porque de todo eso que dijeron, uno ni sabe qué es lo que le podría servir" (GD INFE 5 08042017).

Otro apoyo que puede identificarse en algunos programas de curso es la demanda de lectura previa a la clase, que además de ofrecer la oportunidad de acercar a los estudiantes a los temas propuestos para las sesiones, les permite conocer las maneras en que se presenta el discurso científico en su disciplina.

En ocasiones se pide hacer la lectura con días de antelación y otras, durante la misma clase, como predecesor de una actividad de discusión o de escritura. Usualmente, los profesores sugieren al respecto de este ejercicio la producción de géneros discursivos tales como el resumen o el control de lectura que, por un lado, aproxime al estudiante a la temática por desarrollar, y por el otro, se convierta en insumo para promover la discusión en las clases.

Dicha demanda es asumida por algunos estudiantes como una actividad azarosa, pues para ellos entraña gran dificultad tanto realizar lectura de textos en tiempos determinados, como la socialización o producción de material al final de la sesión. Así puede evidenciarse en la intervención de un informante del grupo de discusión: 
(...) es que si uno no entiende qué es lo que está leyendo, o sea, es decir lo de XXX (un autor que se solicitó leer), ¿cómo voy a escribir una relatoría de algo que nadie entiende? Yo creo que ellos deberían explicar antes de pedir un trabajo escrito (GD INFE 5 08042017).

Cinco profesores ofrecieron explícitamente apoyos a los estudiantes para hacer la lectura en espacios independientes y previos a la clase en la que se discutiría el documento asignado, o durante dicha clase. Por ejemplo, brindaron asesorías presenciales o virtuales, orientaron la lectura en voz alta y discusión de un texto durante la clase, destacando los apartados que consideraban más importantes, explicando o ampliando algunos planteamientos.

Así mismo, algunos docentes entregaron guías para la elaboración de material escrito, en las que ofrecen a los estudiantes orientaciones frente a la tarea que les asignan. No solo entregan derroteros relacionados con la superestructura del texto, sino modelos por seguir para la construcción de diferentes géneros discursivos.

Dichos modelos son entregados por algunos profesores en formatos ampliamente detallados, en los cuales se especifican los apartados que deben constituir el texto solicitado, sus componentes, aspectos formales de producción textual, como las normas APA y algunos ejemplos. Así se identifica en la consigna: "en el material que fue enviado, ahí van a encontrar un modelo del RAE. De ahí pueden sacar el de ustedes tomando como base el otro" (O SG2 15112015)". En otros casos, la entrega de la guía no se realiza a través de un derrotero escrito en físico, sino que se convierte en una sesión de clase magistral en la que se explican pormenores del material que se debe preparar.

Aun así, los estudiantes refieren encontrarse con dificultades para el seguimiento de los parámetros establecidos por el profesor, en tanto, según algunos, las guías están elaboradas en lenguaje técnico que no comprenden o son muy extensas, convirtiéndose para ellos en otra imposición que dificulta el cumplimiento de los objetivos propuestos:

(...) de qué le sirve a uno que le entreguen esa cartilla llena de cosas, de APA, citas, si uno no sabe cómo aplicar, porque a mí nunca me enseñaron a hacer eso, entonces ahora cómo voy a cumplirle al profesor (GD INFE 3 08042017).

Para finalizar, al respecto de la forma en que los estudiantes responden a las demandas académicas propuestas por sus profesores, se encontró que la mayor parte de aquellos, de forma similar a la encontrada por Narvaja de Arnoux et al. (2005), intentan suplir sus necesidades o inquietudes con compañeros de la misma 
Maestría, preferiblemente de su misma línea de investigación, aunque en otros casos también se recurre a amigos o familiares que hayan cursado o se encuentren cursando estudios similares: "yo creo que mi compañera es mi tutora, y yo soy la de ella, para que no nos regañe la profe" (GD INFE 3 08042017).

Los estudiantes solo ocasionalmente recurren a los profesores para aclarar sus dudas, aun cuando se abran espacios tales como asesorías, incluso con los posibles directores de trabajo de grado, asumiéndose por parte de los estudiantes un trabajo en solitario para afrontar las demandas académicas de la Maestría.

\section{Relación entre escritura en los seminarios y proyecto de investigación}

Se identifica una brecha entre las perspectivas de los profesores y de los estudiantes sobre la relación entre la escritura que deben producir estos últimos en los seminarios y la elaboración del proyecto de investigación.

Algunos profesores pretenden que las actividades que proponen en los seminarios a su cargo sean un apoyo para los proyectos de investigación de los estudiantes. Así se percibe en algunos de los microcurrículos:

(...) en la medida del tiempo, en cada sesión se hará un trabajo práctico cuyo propósito es la configuración del anteproyecto de investigación (P SL1).

(...) un ensayo final, que se escribirá y sustentará de manera individual. La escritura del ensayo será la conclusión de un proceso reflexivo e investigativo que los participantes emprenderán a partir del problema investigativo (P SL2).

De manera individual y grupal se analizará los escritos que se lleve al seminario, todo esto con base en los temas que explicará (de manera práctica) el profesor y que se espera sean comprendidos por los estudiantes para que puedan ser aplicados a sus trabajos de investigación (P SE2).

La idea según la cual la elaboración de un género en particular -como los solicitados en los seminarios- aporta a la construcción de otro -como el trabajo de grado- podría estar sustentada en dos presupuestos. Por un lado, que un género complejo se nutre de otros intermedios, lo cual es consistente con la idea según la cual la producción de textos dentro de un género puede mapearse dentro de sistemas de actividad comunales más grandes (Andersen, Bazerman y Schneider, 2014). Por ejemplo, escribir un apartado de un proyecto de investigación, como el marco de referencia conceptual, podría exigir la elaboración de resúmenes, reseñas 
o RAE de los materiales bibliográficos que se leen, así como de planes de texto que ayuden a anticipar la información que se va a incluir y el orden en que se hará.

Por otro lado, el presupuesto de que los aprendices serán capaces de transferir los saberes puestos en juego durante la elaboración de un documento, a la elaboración de otro, pues la transferencia de conocimiento implica la utilización y transposición de conocimientos aprendidos en una situación a una situación nueva (Alexander, 2006). Se asumiría que los temas abordados y los procedimientos puestos en marcha durante la escritura de un texto escrito en los seminarios pueden ser reusados a la hora de enfrentar la escritura de la tesis posteriormente.

No obstante, para los estudiantes no es clara la relación entre los trabajos finales de los seminarios y su proyecto de investigación, hallazgo referido también por Moreno Bayardo (2011). Esta razón explica que esos trabajos sean percibidos por los estudiantes como obligaciones cuyo único propósito es el de complacer al docente:

(...) ahí uno se da cuenta, como que le toca es forzar todo para poder darle gusto al profesor, porque uno de dónde va a meter esas cosas del seminario si... es decir, la investigación no tiene nada que ver con eso (GD INFE 3 08042017).

(...) las tareas que se inventan tienen que ayudarle a ver qué hace con ese proyecto, la tesis. Pero uno por estar pendiente de todo lo demás como que deja eso al otro lado, y ahí sí empiezan a pedir: que no hay avance, que uno es irresponsable (GD INFE 3 08042017).

Más aún, las viven como un esfuerzo innecesario en tanto consideran que todo ejercicio que no se encuentre específicamente orientado a escribir su trabajo de grado no es de utilidad: "y para qué todo eso, si al final el cartón se lo entregan cuando haga la tesis, eso es lo único que piden" (GD INFE 2 08042017).

Durante algunas clases los profesores abordan temáticas generales del campo de la educación con las que buscan aproximarse a todas las propuestas de investigación de los estudiantes, o destinan segmentos de la clase a discutir alguna propuesta de investigación de un maestrante en particular. Sin embargo, ninguno de los dos esfuerzos es valorado positivamente por estudiantes participantes en el grupo de discusión:

(...) o sea... uno no dice que se las sabe todas, pero algo ya traía sobre el campo educativo, las teorías, las leyes, y además de la experiencia... Pero 
¿investigar?, de eso sí poco, pues a mí nadie me ha enseñado (GD INFE 2 08042017).

(...) a mí me gustaba cuando me hablaban de lo que era mío, de lo que yo sabía. Pero y cuando empezaban a explicarle a los demás, o sea, no es por ser egoísta ni nada, pero es que ese era mi tiempo que se perdía, el de todos, pues menos de la persona a la que le estaban ayudando (GD INFE 4 08042017).

La primera cita revela que la discusión de temas educativos en clase es percibida como impertinente con miras a realizar su trabajo de grado, ante la sentida necesidad de recibir apoyo para la formación investigativa. Parece que el estudiante no logra identificar relación entre ambos asuntos, aunque claramente el programa académico que cursan les exige desarrollar una propuesta de investigación en educación. La segunda cita, por su parte, da cuenta de la incapacidad de asumir la reflexión de un compañero guiada por el docente en presencia del grupo, como soporte al propio aprendizaje. Las intenciones docentes, entonces, no encuentran eco en las interpretaciones de los maestrantes.

\section{CONCLUSIONES}

El desarrollo de esta investigación nos permitió avanzar en la comprensión de las prácticas de escritura que tienen lugar en un Programa de Maestría en Educación con énfasis en investigación y de la manera en que los protagonistas, docentes y estudiantes, las interpretan.

Elementos de la estructura curricular y de los programas de seminario, así como la observación de clases, muestran una alta presencia de la escritura a lo largo del proceso de formación y la intención docente de apoyar a los estudiantes en las tareas que la implican. No obstante, algunas actuaciones como comentar los textos de los aprendices, que los profesores realizan con la intención de contribuir a su formación y a la cualificación de sus textos, son percibidas por los maestrantes como el deseo de entorpecer su avance en el proyecto de investigación. Paradójicamente, estos también valoran negativamente no obtener realimentación de sus trabajos escritos, pues asumen que no son siquiera leídos por sus profesores, percepción compartida y criticada por el docente entrevistado.

Los hallazgos revelan también que no siempre se ejecuta lo planeado, en cuanto a los géneros y prácticas de escritura que se espera introducir en las sesiones de clase. Aunque es comprensible ajustar lo planeado de acuerdo con el 
desarrollo de un curso, habría que valorar si los cambios implementados permiten alcanzar los objetivos de aprendizaje trazados para ese espacio académico.

Además, se identificó que existe tensión entre las voces de los estudiantes y las de los profesores con respecto a la relación entre la escritura en los seminarios y la elaboración del trabajo de grado, pues a pesar de las intenciones de los docentes, los maestrantes no comprenden la relación entre ambas tareas.

Parece necesario el estudio y la discusión de esta situación en el interior del cuerpo docente y administrativo del programa, de modo que puedan, si lo consideran deseable, tejer puentes entre la escritura en los seminarios y la escritura del trabajo de grado, de maneras comprensibles y rastreables para ambos protagonistas.

La visibilización de las tensiones entre lo propuesto y lo ejecutado, entre las intenciones docentes y las interpretaciones estudiantiles, entre el ideal de formar a investigadores y el de formar a expertos en educación, y entre la escritura en los seminarios y el trabajo de grado puede alentar procesos de reflexión orientados a la toma de decisiones pedagógicas y didácticas en el interior del Programa analizado y de otros con características similares. Por ejemplo, discutir las relaciones entre escritura, investigación y aprendizaje podría animar a los profesores a acompañar, al interior de los seminarios, la producción de trabajos escritos que aporten a la formación de los estudiantes como investigadores o como expertos en su campo disciplinario y como escritores académicos o científicos, explicitando y discutiendo sus intenciones con los aprendices.

También esperamos que estos hallazgos contribuyan a avanzar en el campo disciplinario de los estudios sobre escritura en educación superior, pues aportan información sobre las prácticas de escritura en un espacio de formación y un campo disciplinario particular en el nivel de posgrado, que ha sido escasamente investigado en la región. Son bienvenidos estudios que exploren y acompañen los esfuerzos de los programas académicos por transformar las prácticas docentes en beneficio de la formación escritural de los estudiantes.

\section{REFERENCIAS}

Aguilar, L. E., y Fregoso, G. (2013). La literacidad académica en estudiantes de posgrado: límites y principios de una práctica académica. En Memoria de trabajos del XII Congreso Latinoamericano para el desarrollo de la lectura y la escritura (pp. 827-834). Recuperado de https://www.inaoep.mx/ cplorg/pdfs/investigacion_editorial/m1.pdf. 
Alexander, P. A. (2006). Psychology in Learning and Instruction. Nueva Jersey: Pearson.

Álvarez, G., y Difabio de Anglat, H. (2017). La actividad metalingüística en espacios de interacción entre pares: reflexiones en torno a un taller virtual orientado a la escritura de la tesis de posgrado. Perfiles Educativos, 39(155), 5167.

Amieva, R., y De Angelo, C. (2010). La enseñanza de la escritura académicocientífica para la pronta definición de los proyectos de tesis de posgrado en ingería. En A. Vásquez, M. Novo, I. Jakob y L. Pelliza (Comps.), Lectura, escritura y aprendizaje disciplinar (pp. 785-791). Recuperado de https://www.unrc.edu.ar/unrc/digtal/libro_jornadas_unesco_unrc_2010.pdf.

Andersen, J., Bazerman, C., y Schneider, J. (2014). Beyond single genres: Pattern mapping in global communication. En E. M. Jakobs y D. Perrin (Eds.), Handbook of Writing and Text Production (pp. 305-322). Berlin: De Gruyter Mouton.

Bajtín, M. M. (1982). El problema de los géneros discursivos. En Estética de la creación verbal (pp. 248-293). Coyoacán, México: Siglo XXI editores.

Barton, D., y Hamilton, M. (2004). La literacidad entendida como práctica social. En

143 V. Zavala, M. Niño-Murcia y P. Ames (Eds.), Escritura y sociedad. Nuevas perspectivas teóricas y etnográficas (pp. 109-139). Recuperado de https://www.academia.edu/13796818/Escritura_y_sociedad._Nuevas_perspec tivas_te\%C3\%B3ricas_y_etnogr\%C3\%A1ficas.

Carlino, P. (2005). La escritura en la investigación. Conferencia presentada en el Seminario permanente de investigación de la Maestría en Educación de la UdeSA, Buenos Aires, Argentina. Recuperado de http://www.aacademica.org/instituto.de.lingoistica.de.la.universidad.de.buen os.aires/box/paula.carlino/66.pdf.

Carlino, P. (2009). Exploración de géneros, diario de tesis y revisión entre pares: análisis de un ciclo de investigación-acción en talleres de tesis de posgrado. En E. Narvaja de Arnoux (Ed.), Escritura y producción de conocimiento en las carreras de posgrado (pp. 227-246). Buenos Aires, Argentina: Santiago Arcos.

Carrasco, A., Kent, R., y Keranen, N. (2012). Learning Careers and Enculturation: Production of Scientific Papers by PhD Students in a Mexican Physiology Laboratory: An Exploratory Case Study. En C. Bazerman, C. Dean, J. Early, K. Lunsford, S. Null, P. Rogers y A. Stansell (Eds.), International Advances in Writing Research: Cultures, Places, Measures (pp. 335-351). Recuperado de: https://wac.colostate.edu/books/perspectives/wrab2011/.

Cassany, D., y Morales, Ó. (2009). Leer y escribir en la universidad: los géneros científicos. En D. Cassany (Comp.), Para ser letrados. Voces y miradas sobre la lectura (pp. 109-128). Barcelona, España: Paidós. 
Castelló, M., González, D., e Iñesta, A. (2010). La regulación de la escritura académica en el doctorado: el impacto de la revisión colaborativa en los textos. Revista española de pedagogía, 68(247), 521-537.

Chois, P., y Jaramillo, L. (2016). La investigación sobre la escritura en posgrado: estado del arte. Lenguaje, 44(2), 227-259. doi: 10.25100/lenguaje.v44i2.4622

Colombo, L. (2012a). Escritura de posgrado y aprendizaje Situado. Trabajo presentado en el IV Congreso Internacional de Investigación y Práctica Profesional en Psicología XIX Jornadas de Investigación VIII Encuentro de Investigadores en Psicología del MERCOSUR, Buenos Aires, Argentina. Recuperado de https://www.aacademica.org/000-072/431.pdf.

Colombo, L. (2012b). Grupos de escritura en el posgrado. Trabajo presentado en VIII jornadas de material didáctico y experiencias innovadoras en educación superior, Buenos Aires, Argentina. Recuperado de https://www.academia.edu/13187476/Grupos_de_escritura_en_el_posgrado.

Colombo, L. (2016). Ayudas pedagógicas para la revisión de la literatura en posgrado. En G. Bañales, M. Castelló y N. A. Vega (Coords.), Enseñar a leer y escribir en investigación. Propuestas educativas basadas en la investigación (pp. 259280). Recuperado de http://www.fundacionsm.org.mx/sites/default/files/Ense\%C3\%B1ar\%20a\%20leer\%20y\%20escribir.p df.

Colombo, L. (2017). Los grupos de escritura y el aprendizaje situado en el posgrado.Jornaler@s Revista científica de estudios literarios y lingüísticos, 3(3), 154-164.

Colombo, L., y Zambrano, J. (2013). ¿Qué escriben los estudiantes de posgrado? Tipos de textos solicitados en seminarios. Forma y Función, 26(2), 41-55.

Corbetta, P. (2003). Metodología y técnicas de investigación social. Madrid, España: McGraw Hill.

Cruz Martínez, A. (2014). La atención de la escritura académica en el posgrado: una experiencia en la UPN. Revista de Investigación educativa de la REDIECH, 5(9), 51-59.

Day, R. A. (2005). Cómo escribir y publicar trabajos científicos. Washington: Organización Panamericana de la Salud.

Delamont, S., y Atkinson, P. (2001). Doctoring Uncertainty: Mastering Craft Knowledge. Social Studies of Science, 31(1), 87-107.

Fernández, L., y Guevara, J. (2017). Los talleres de tesis como aproximación a una comunidad de práctica. Cuadernos de Investigación Educativa, 8(1), 31-46.

Gaviria, Y., Rincón, N., y Triana, M. (2016). Estado del arte: Prácticas de formación en investigación del profesor investigador a nivel posgradual en educación (Tesis de maestría). Universidad Santo Tomás, Bogotá, Colombia. 
Goetz, J. P., y LeCompte, M. D. (1988). Etnografía y diseño cualitativo en investigación educativa. Madrid, España: Ediciones Morata.

Guerrero, R., y Mostacero, R. (2014). Logros y alcances de escribir el trabajo de grado en un grupo de escritura. Educere, 18(61), 527-534.

Hernández Zamora, G. (2009). Escritura académica y formación de maestros ¿Por qué no acaban la tesis? Tiempo de educar, 10(19), 11-40.

Horowitz, D. (1986). Process, Not Product: Less Than Meets the Eye. TESOL Quarterly, 20(1), 141-144.

Miller, C. (1984). Genre as social action. Quarterly Journal of Speech, 70(2), 151-167. doi: https://doi.org/10.1080/00335638409383686

Maestría en Educación (2007). Documento presentado al Ministerio de Educación Nacional para obtener registro calificado. Universidad del Cauca. Facultad de Ciencias Exactas, Naturales y de la Educación, Popayán, Colombia.

Ministerio de Educación Nacional. (22 de enero de 1980). Decreto 80 de 1980. Diario Oficial N. 35.465. Recuperado de https://www.mineducacion.gov.co/1759/w3-article102556.html?_noredirect $=1$.

Ministerio de Educación Nacional. (03 de abril de 2006). Decreto 1001. Diario Oficial N. $\quad 46230.4$ Recuperado de http://www.mineducacion.gov.co/1621/articles-96961_archivo_pdf.pdf.

Miras, M. y Solé, I. (2007). La elaboración del conocimiento científico y académico. En M. Castelló (Coord.), Escribir y comunicarse en contextos científicos y académicos: conocimientos y estrategias (pp. 83-112). Barcelona, España: Graó.

Moreno Bayardo, M. G. (2011). La formación de investigadores como elemento para la consolidación de la investigación en la universidad. Revista de la Educación Superior, 40(158), 59-78.

Mostacero, R. (2013). Construcción de la reseña crítica mediante estrategias metacognitivas. Lenguaje, 41(1), 169-200. doi: 10.25100/lenguaje.v41i1.4966

Narvaja de Arnoux, E. (2010). La escritura de tesis: apoyos institucionales y propuestas pedagógicas. En A. Vásquez, M. Novo, I. Jakob y L. Pelliza (Comps.), Lectura, escritura y aprendizaje disciplinar (pp. 25-40). Recuperado de https://www.unrc.edu.ar/unrc/digtal/libro_jornadas_unesco_unrc_2010.pdf.

Narvaja de Arnoux, E., Borsinger, A., Carlino, P., Di Stefano, M., Pereira, C., y Silvestre, A. (2005). La intervención pedagógica en la escritura de tesis de posgrado. Revista de la Maestría en Salud Pública, 3(6). Recuperado de https://media.utp.edu.co/referencias-

bibliograficas/uploads/referencias/articulo/89-la-intervencin-pedaggica-en-elproceso-de-escritura-de-tesis-de-postgradopdf-0j19S-articulo.pdf. 
Pereira, C., y Di Stefano, M. (2007). El taller de escritura en posgrado: Representaciones sociales e interacción entre pares. Revista Signos, 40(64), 405430. Recuperado de http://www.redalyc.org/articulo.oa?id=157013771007.

Pereyra, D., y Cardoso, N. (2017). Reflexiones sobre el dictado de talleres de escritura en el posgrado. El contexto de producción de una tesis. Revista Docência do Ensino Superior, 7(1), 57-73.

Russell, D. R., y Cortes, V. (2012). Academic and scientific texts: The same or different communities? En M. Castelló y C. Donahue (Eds.), University writing: selves and texts in academic societies (pp. 3-18). Bingley, Inglaterra: Emerald.

Sabaj, O. (2009). Descubriendo algunos problemas en la redacción de Artículos de Investigación Científica (AIC) de alumnos de postgrado. Revista Signos, 42(69), 107-127. Recuperado de http://www.scielo.cl/pdf/signos/v42n69/a06.pdf.

Scardamalia, M., y Bereiter, C. (1992). Dos modelos explicativos de los procesos de composición escrita. Infancia y Aprendizaje, 58, 43-64.

Swales, J. M. (1990). Genre Analysis: English in academic and research settings. Cambridge: Cambridge University Press.

\section{SOBRE LOS AUTORES}

\section{Héctor Iván Guerrero}

Fonoaudiólogo. Magíster en Educación-Línea de investigación en Pedagogía de la Lectura y la Escritura. Investigador en Escritura académica en posgrado, Fonoaudiología, Pedagogía de la lectura y la escritura, Enseñanza y aprendizaje de Lengua extranjera e Inclusión Educativa. Actualmente se desempeña como Profesional de Apoyo en Inclusión en la Fundación Centros de Aprendizaje Neuroharte, Popayán, Colombia

Correo electrónico: higuerrero@unicauca.edu.co

\section{Pilar Mirely Chois Lenis}

Fonoaudióloga. Especialista en la enseñanza de la lectura y la escritura en lengua materna. Magíster en Lingüística y español. Candidata a Doctora en Ciencias de la Educación. Elabora actualmente su tesis doctoral Prácticas docentes sobre la escritura en espacios de formación en investigación de posgrados en Salud en dos universidades del suroccidente colombiano. Docente titular Universidad del Cauca, Popayán, Colombia. Investigadora en prácticas pedagógicas sobre la escritura en grados iniciales y en la educación superior.

Correo electrónico: pilarchois@unicauca.edu.co 